\title{
In-situ detection of single particle impact, erosion/corrosion and surface roughening
}

\author{
P. R. Birkin*a, R. Lear ${ }^{a}$, L. Webster ${ }^{\text {a }}$, L. Powell ${ }^{a}$ and H. L. Martin ${ }^{a}$
}

\begin{abstract}
Particle impact is technologically important and can cause significant damage to a surface. Detecting the approach and impact of a particle would give key information on the process. High-speed imaging of this process gives information on a particle's velocity and movement but does not answer key questions relating to changes in electrochemical properties of a surface caused by the resultant damage on impact. Furthermore, it is difficult to apply in non-transparent media. To gain this key information, we have deployed a high-speed electrochemical impedance technique with the ability to determine the uncompensated resistance, Faradaic current and effective capacitance of an electrode. This technique has a time resolution of $1.25 \mu \mathrm{s}$. Individual impacts of sand particles in a fluid jet (jet velocity $\sim 4-5 \mathrm{~m} \mathrm{~s}^{-1}$ ) are used to cause erosion/corrosion of an aluminium interface. Surface properties are shown to change after individual particle impacts, which is preceded by the electrochemical detection of the particle as it approached the solid/liquid interface. For the first time, the in-situ roughening of the electrode surface is reported for a single particle impact. A link between the effective mass loss of the electrode and the overall surface erosion is shown, with an equivalent roughening rate of $8.5 \mathrm{~F} \mathrm{~g}^{-1}$ as determined from the data. This study shows how individual sand particles, and the damage they cause to an interface, can be detected with high precision and new insight. This will improve our understanding of erosive environments.
\end{abstract}

Keywords: particle, impact, detection, impedance, roughening, imaging

\author{
*Corresponding author \\ prb2@soton.ac.uk \\ ${ }^{a}$ Chemistry, University of Southampton, Highfield. Southampton, SO171BJ, UK
}




\subsection{Introduction}

Particles play a key role in many technological advancements, from catalysis to power source development. In addition, particles are also thought to have significant impact on human health. Hence, the characterisation of particles and their interactions with surfaces is important in understanding their physical and chemical effects. One such interaction is the effect that a fast-moving particle can have on the structure of an interface[1]. Erosion[2,3] of solid materials, in the gas or liquid phase, can be observed to have profound effects on material structure and the performance of a system $[4,5]$. In turn, the investigation of these erosion/corrosion events has been undertaken in a multidisciplinary effort to understand the underlying mechanisms and to characterise a particular material in harsh erosive environments[6]. As part of these investigations, the study of passive electrode interfaces exposed to an erosive event is of interest. Examples of these environments include cavitation[7-11] and particle impingement[12-14]. In many cases, the effect of the erosive mechanism on the surface has been quantified ex situ. However, it is possible to follow these processes in situ. A good example is the monitoring of the erosion and corrosion of an electrode exposed to cavitation[11,15]. In essence, the electrode is passive unless a sufficiently energetic process removes some of the protective layer. At this point, the surface repassivates which is accompanied by an anodic current transient $[10,11,14,15]$. Hence, it is the post erosion or 'healing process' which is recorded. Although useful this does not give any other information on the event that caused the erosion itself. To circumvent this limitation, a technique based on high-speed impedance[16,17] analysis has been developed[13,18]. In this case, the uncompensated resistance and the effective capacitance of the electrode (through the measurement of the electrode impedance), was acquired with a $2 \mu$ s resolution throughout either cavitation erosion (via bubble dynamics) or sand particle impact[13]. These studies showed that in the former case, bubble growth and collapse preceded the erosion/corrosion event[18] while in the latter case particle approach and impact as well as tumbling[13] could be detected prior to the erosion/corrosion process. These observations are powerful in that the full mechanism can be probed rather than just the aftereffects on their own. Some issues remain. First, although the resolution of $2 \mu \mathrm{s}$ is useful, increasing this time resolution is desirable to detect short lived or multiple events. Second, limitations associated with the instrumentation meant that a small background current was observed (as opposed to zero under non-erosive conditions). These limitations are removed here with improved temporal resolution and improved instrumentation. In this investigation, sand particles entrapped in a fluid jet impinge on the surface of an aluminium electrode causing erosion and 


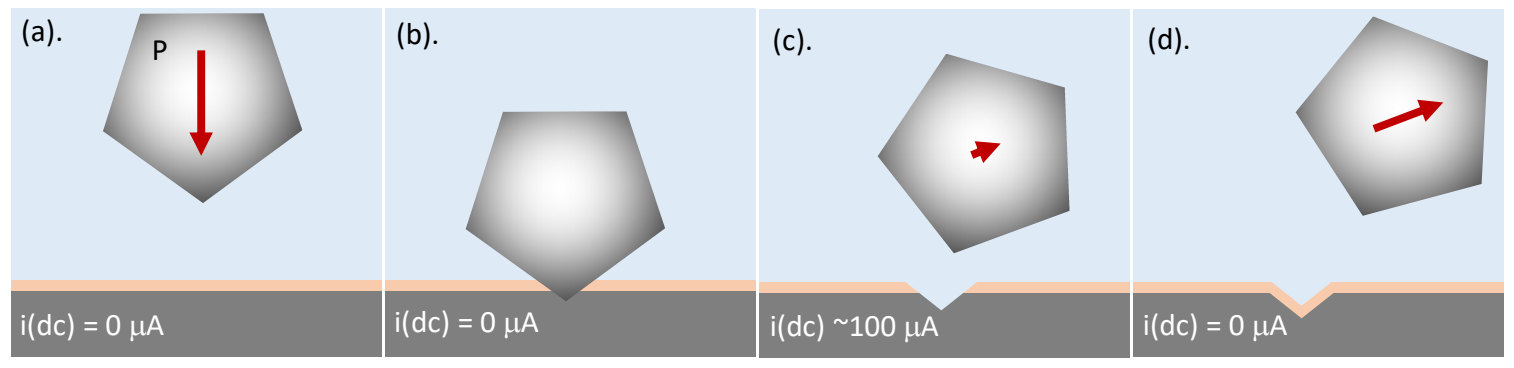

Figure 1. Schematic showing the approach of a particle $(P)$ to an interface. (a) shows the oxide ( ) covered electrode surface ( ) and the particle approaching from the jet. (b) shows the impact event which damages the surface and the passive oxide layer.(c) shows the exposed metal as it corrodes which is accompanied with a current $(\mathrm{i}(\mathrm{dc}))$. (c) shows the oxide layer passivated to "heal" the exposed aluminium but with an increased surface area through the roughening process. The red arrows $(\rightarrow)$ show the magnitude and direction of motion of the particle. The time progress from left to right ((a) to (d)). Note, not to scale.

reformation of the oxide layer. This system was chosen as sand particles are a common erosive element and the corrosion transient produced through erosion of the oxide layer on aluminium is dynamic, requiring high temporal resolution approaches.

Figure 1 shows a schematic representation of an individual particle impact onto a passive electrode surface. This highlights the stages of the process (from (a) to (d)) and the individual events that will be investigated. Note, in the technique presented here, the high-speed impedance measurement will detect the approach, impact and departure (figure 1 (a)-(d)) as well as the surface damage (c) as a result of the individual impact.

Importantly, for the first time, this surface damage or roughening is measured in situ (and at the point of impact) as single particle impacts occur. Finally, a correlation between erosive damage and changes to the electrode surface area as determined from the electrochemical impedance data is presented.

\subsection{Materials and Methods}

A jet system was constructed from a pump connected to a $\sim 1 \mathrm{~mm}$ diameter glass orifice. Fluid (an electrolyte) was pumped through the system at velocities in the range of 4-5 $\mathrm{m} \mathrm{s}^{-1}$ (determined by measuring the mass of fluid ejected from the jet over a fixed time and verified by monitoring the particle velocity as it left the jet with a high-speed camera). The jet was directed onto an electrode substrate which consisted of an electrode (aluminium, $100 \mu \mathrm{m}$ diameter) made by sealing a microwire (Advent Research Materials, Oxford, UK) in an epoxy resin (Epofix, Struers, Cleveland, USA) within a stainless-steel tube (Coopers Needle Works Ltd., $6 \mathrm{~mm}$ OD, Birmingham, UK). This stainless-steel (SS) tube acted as the counter/reference 
electrode and a support body for the working electrode. The electrode was polished to a mirror like finish ( $0.3 \mu \mathrm{m} \mathrm{Al}_{2} \mathrm{O}_{3}$, Struers on Struers microcloth) prior to experiments.

The jet was aligned to the centre of the electrode using two micropositioners (Zaber, Vancouver, Canada) to control movement in the XY plane and a manual micrometre and stage (Thor labs, Newton, USA) to control movement in the Z plane (see figure legends for electrode to jet $Z$ separation). A schematic of the apparatus employed is shown in Figure S1. The precise alignment of the jet was identified through monitoring of the uncompensated resistance of the electrode, which increased as the jet passed over the surface of the active element (in this case the jet was positioned close to the interface so that the tip was easily detectable in the impedance data). A pump circulated electrolyte from the main reservoir through the jet. Particles (sand pre-filtered through an aperture of the same size as employed in the jet) were added to the flow system via a loading port situated between the pump and the jet. Typically these sand particle are non-spherical and have approximate diameters centred around 250 $\mu \mathrm{m}[13]$. To determine the electrochemical characteristics of the electrode as a particle approaches/impacts, a high-speed impedance approach was adopted. This has been described in detail elsewhere[18]. However, in the experimental setup deployed here the impedance and phase angle between the voltage and current response of the electrode was determined as a function of time (with a $1.25 \mu$ s resolution). This required recording the data at a sample rate of $8 \mathrm{MHz}$. The current and voltage data was recorded using a PCI-DAS4020/12 DAQ card (Measurement Computing, Norton, USA) and then post processed (using VB6 or VS2010 and Measurement Studio, NI, Austin, USA, see ref[18]). The current follower consisted of a fixed gain $\left(10^{4} \vee A^{-1}\right)$ system based on an ADA4891 operational amplifier from Analogue devices (Norwood, USA). The potential of the working electrode was maintained at $0 \mathrm{~V} v s$. the SS reference. An AC perturbation ( $800 \mathrm{kHz}$, typically $200 \mathrm{mV}$ peak-to-peak amplitude) was applied using a function generator (TTi, TG1010A, Cambridgeshire, UK). The actual applied wave form was recorded in each data set along with the current response of the electrode. Care was taken to ensure that the conditions (e.g. the electrode size, material and electrolyte conductivity[19]) were conducive to the accurate estimation of both the uncompensated resistance and the inferred electrode capacitance. In this respect a phase angle between the current and applied voltage perturbation, which is not, extreme (e.g. not close to $0^{\circ}$ or $90^{\circ}$ ) is desirable. This allows for an acceptable error in both the uncompensated resistance and the effective capacitance to be determined. The full description of this consideration is contained within the SI information. The data gathered (the voltage time and the current time histories) are processed using a DSP approach. In this system a set of points are taken and analysed to 
determine the magnitudes and phase relationship between the voltage and current signals. These values could then be used to determine the impedance and in turn the values of the uncompensated resistance and the effective capacitance. To do this an appropriate simple model of the electrode (a capacitor and resistor in series) was employed. More details can be found of the approach elsewhere[18]. High-speed camera images were recorded using a Fastcam-APX RS from Photron (Tokyo, Japan). A 12X Navitar lens with a variable 0.58X-7X zoom and a .67X adaptor tube was fitted onto the camera unit. The imaging data were analysed using Photron FASTCAM Viewer ver. 4.0.1.0 software. The data acquisition of the high-speed camera and the electrochemical impedance system was initiated by a common trigger, which was the signal from a DSO (Owon, SDS7102V, Zhangzhou, China).

All solutions for impact experiments consisted of $0.5 \mathrm{~mol} \mathrm{dm}^{-3} \mathrm{Na}_{2} \mathrm{SO}_{4}$ in aerobic purified water $\left(20-25^{\circ} \mathrm{C}\right.$ ) from a from a Suez (Thame, UK) Select Fusion water system $(18.2 \mathrm{M} \Omega \mathrm{cm})$. Note the density and viscosity of $\mathrm{Na}_{2} \mathrm{SO}_{4}$ solutions have been reported previously under a variety of conditions $[20,21]$.

\subsection{Results}

Once a data set had been captured from the jet impingement experiment, it was processed to extract the relevant electrochemical data. Figure 2 shows the uncompensated resistance[22] $(\mathrm{Ru})$, capacitance $(\mathrm{C}(\mathrm{av}))$, faradaic current $(\mathrm{i}(\mathrm{dc}))$ and an estimation of the repassivation mass loss associated with passive film reformation. In this plot, the capacitance data has had a 10point moving average applied. Figure S5 shows an example of the unaveraged data in comparison with the averaged data for reference. It was found that this was only necessary for the capacitance data as the noise on the phase component (and its propagation), resulted in a noisy capacitance signal (see SI for further discussion). The repassivation mass refers to the reformation of $\mathrm{Al}_{2} \mathrm{O}_{3}$. In order to calculate this mass, the $\mathrm{i}(\mathrm{dc})$ current was integrated above a threshold of $0.7 \mu \mathrm{A}$ (determined to avoid unwanted noise contributing to the calculation). The resultant charge was then used to calculate the mass of $\mathrm{Al}_{2} \mathrm{O}_{3}$ produced under these conditions. Note, this calculation assumes that the charge measured through this integration is converted to a mass associated with the $\mathrm{Al}_{2} \mathrm{O}_{3}$ produced to 'heal' the damaged area. In addition, other processes (such as other reactions or double layer charging) are ignored at this time. This means that the mass calculated in this fashion represents an upper limit. Figure 2 shows four erosion events in the $15 \mathrm{~ms}$ time period shown (each marked with a '*' on the i(dc) data). Each of the erosion transients is accompanied with a change in the uncompensated resistance and the implied capacitance of the electrode. As the sand particle approaches the 
electrode the uncompensated resistance increases[22] and the capacitance falls. However, the model used to interpret the data is a simple resistor and capacitor (to represent the capacitance of the electrode interface) in series and does not account for more complex electrochemical scenarios (such as constant phase elements[23] and other interactions that may cause the capacitance to be related to the uncompensated resistance). Nevertheless, the data shows the presence of these insulating particles as clear changes in uncompensated resistance and effective capacitance, the magnitude of which can be related to the size and movement of the particle near the electrode surface. Figure 2 also shows that the number of transient erosion/corrosion events is a small subset of the total number of transients detected by the system (via changes in the uncompensated resistance, for example). For example, there are $\sim 100$ erosion/corrosion events detected in the $625 \mathrm{~ms}$ data window while there are $\sim 400$ uncompensated resistance transients. This suggests that the number of particles that come close to the Al electrode, or have insufficient energy on impact to damage the passive layer, is larger than the number of events that cause surface erosion. These data runs were repeated many times $(>10)$. In each case a significant number of these electrode particle encounter

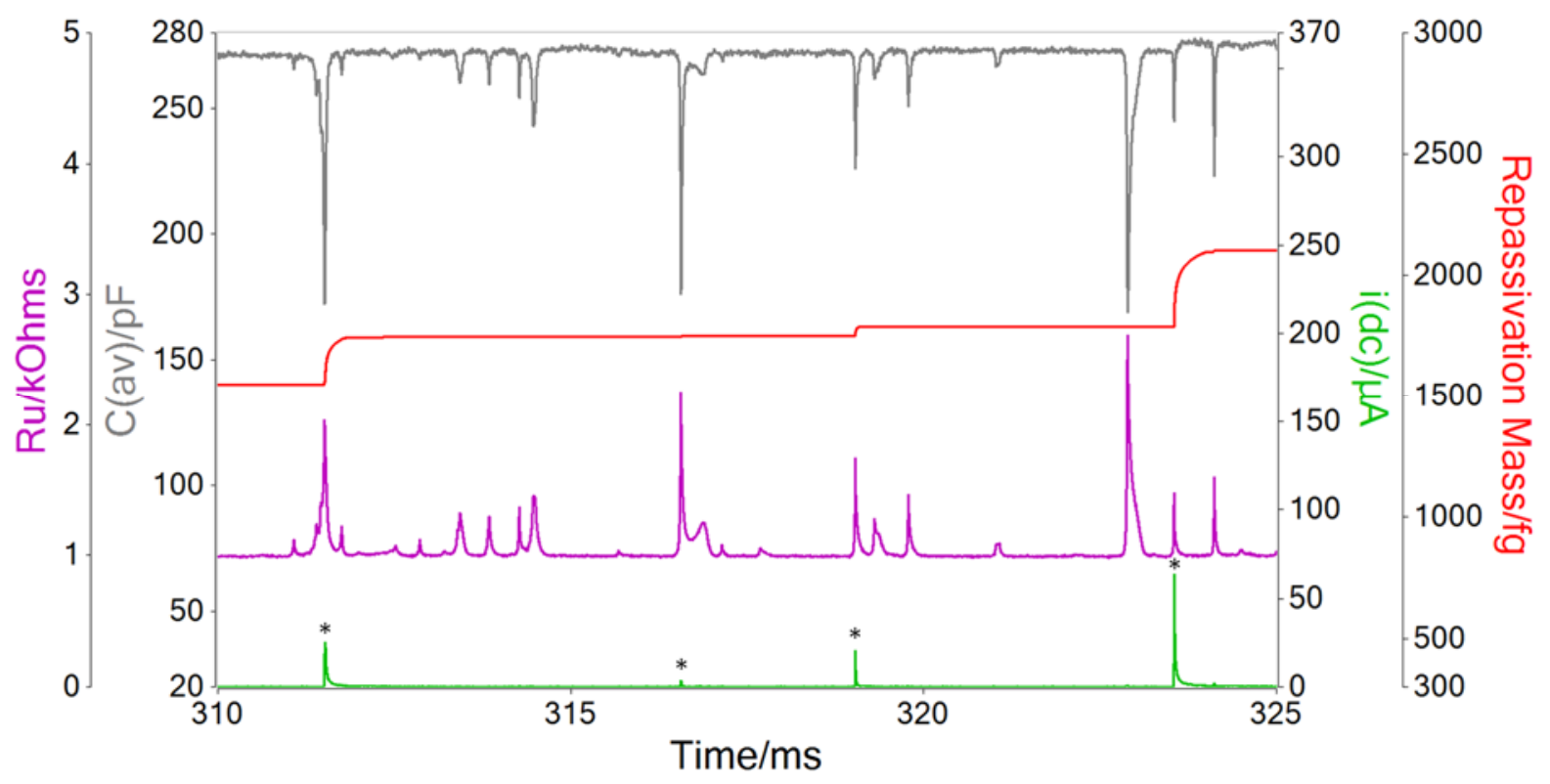

Figure 2. Plot showing the uncompensated resistance $(\mathrm{Ru},-)$, capacitance (with a moving point average of 10 points, $\mathrm{C}(\mathrm{av}),-)$, the faradaic current $(\mathrm{i}(\mathrm{dc}),-)$ and the mass change $(-)$ for a $100 \mu \mathrm{m}$ diameter Al electrode, (held at $0 \mathrm{~V}$ vs. SS) exposed to sand particle impact in a recirculating aerobic electrolyte (0.5 $\left.\mathrm{M} \mathrm{Na}_{2} \mathrm{SO}_{4}\right)$ at room temperature. An $800 \mathrm{KHz}\left(200 \mathrm{mV}_{\mathrm{pp}}\right)$ wave was applied to the system. The jet was positioned centrally over the Al electrode in the $X Y$ plane, above the electrode, and elevated to $2.0 \mathrm{~mm}$ in the $\mathrm{Z}$ axis. The ${ }^{\prime * \prime}$ indicates particle impacts that caused a detectable erosion/corrosion event. The 'Repassivation Mass' axis refers to integration of the $\mathrm{i}(\mathrm{dc})$ signal above $0.7 \mu \mathrm{A}$ and conversion using Faraday's law for the generation of $\mathrm{Al}_{2} \mathrm{O}_{3}$. events could be detected (many 100s). 
Figure 3 shows an expanded section of the data centred on an event that caused significant erosion at $\sim 0.3235 \mathrm{~s}$.

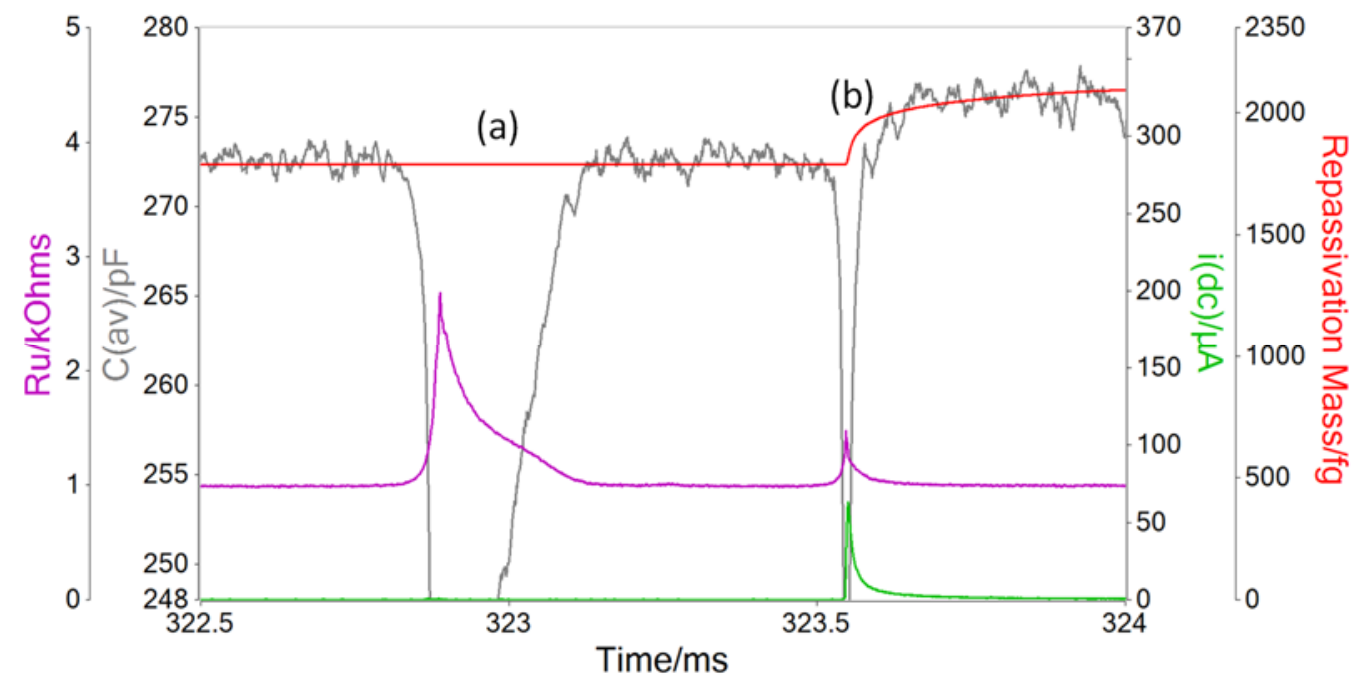

Figure 3. Plot showing an expanded region of the data shown in figure 1 and annotated to show two events ('(a)' and '(b)'). All conditions are as in figure 1.

This event (labelled ' $(\mathrm{b})^{\prime}$ ) produced an erosion/corrosion transient maximum of $63 \mu \mathrm{A}$. However, the transients in the uncompensated resistance and capacitance suggest that this event is the smaller of the two shown or that the orientation of the particle/electrode encounter produces a smaller perturbation in the electrochemical impedance data. The data also shows that the capacitance of the electrode before and after event '(b)' caused a change in the electrode capacitance (under these conditions). This suggests that the surface of the electrode was altered by the impact and it is likely that some form of surface roughening was produced in this case. Event ' $(b)^{\prime}$ ' caused a change in capacitance of $\sim 4 \mathrm{pF}$. If it is assumed that this capacitance change was caused by roughening of the interface (through indentation, for example), the area increase as a result of this impact was $\sim 1.26 \times 10^{-6} \mathrm{~cm}^{2}$. This is $\sim 2$ orders of magnitude less that indentations from single sphere impact experiments[24]. Figure 3 also shows that another event (labelled ' $(a)^{\prime}$ ) caused little or no perceivable erosion of the surface and, at the same time, left the capacitance of the electrode unaltered. Figure 3 also shows that the mass change (associated with the anodic faradaic signal) is significant at $\sim 300$ fg for this single event ('(b)').

Figure 4 shows the change in the capacitance of the electrode surface and the associated mass change calculated by integration of the faradaic current over the complete data run (as opposed to ex situ measurements[23,25]). It appears that there is a strong association between changes in the capacitance of the electrode and the regenerated mass on the surface. This is attributed to the changes in the surface roughness over the exposure period as the 
particles hit the surface and damage the interface (see figure 1). The rate at which this surface damage occurs is of interest, as it will relate to the ability of a surface to withstand damage caused by the erosion mechanism. Figure 5 shows a plot of the estimated mass change plotted as a function of the electrode capacitance. In this case, the horizontal striations on the plot show the data when particle/electrode encounters occurred.

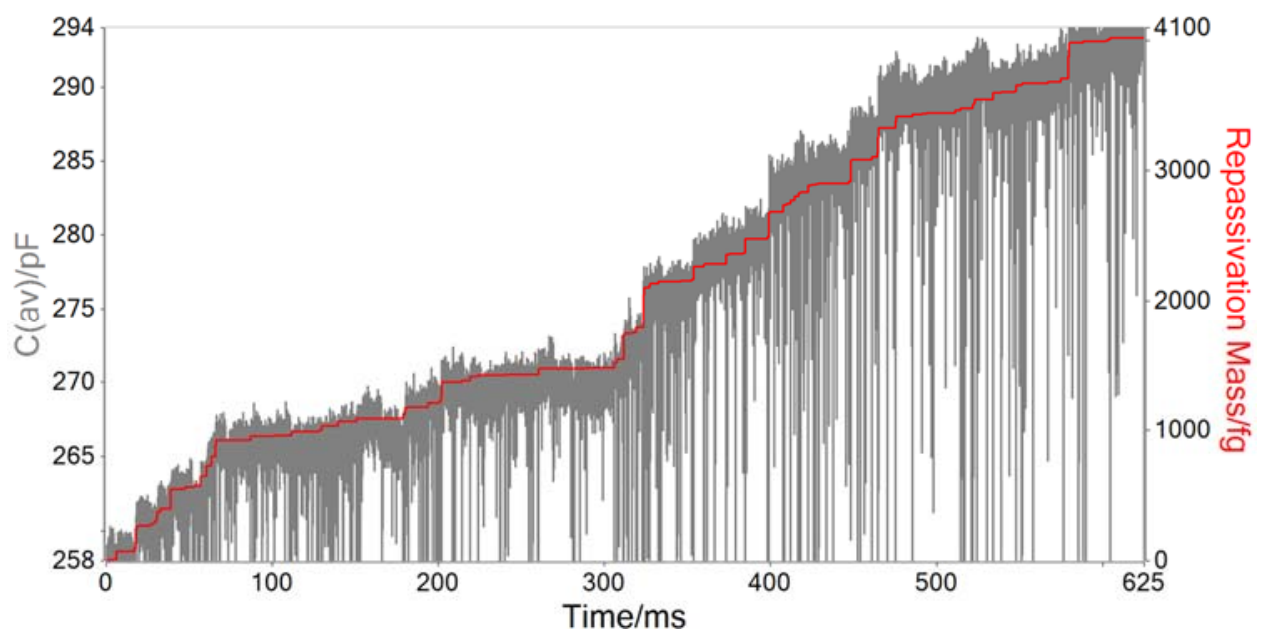

Figure 4. Plot showing the capacitance ( - , with a moving point average of 10 points, $C(\mathrm{av})$ ) and the regenerated mass $(-)$ for a $100 \mu \mathrm{m}$ diameter $\mathrm{Al}$ electrode over the complete run. All conditions are as in figure 1.

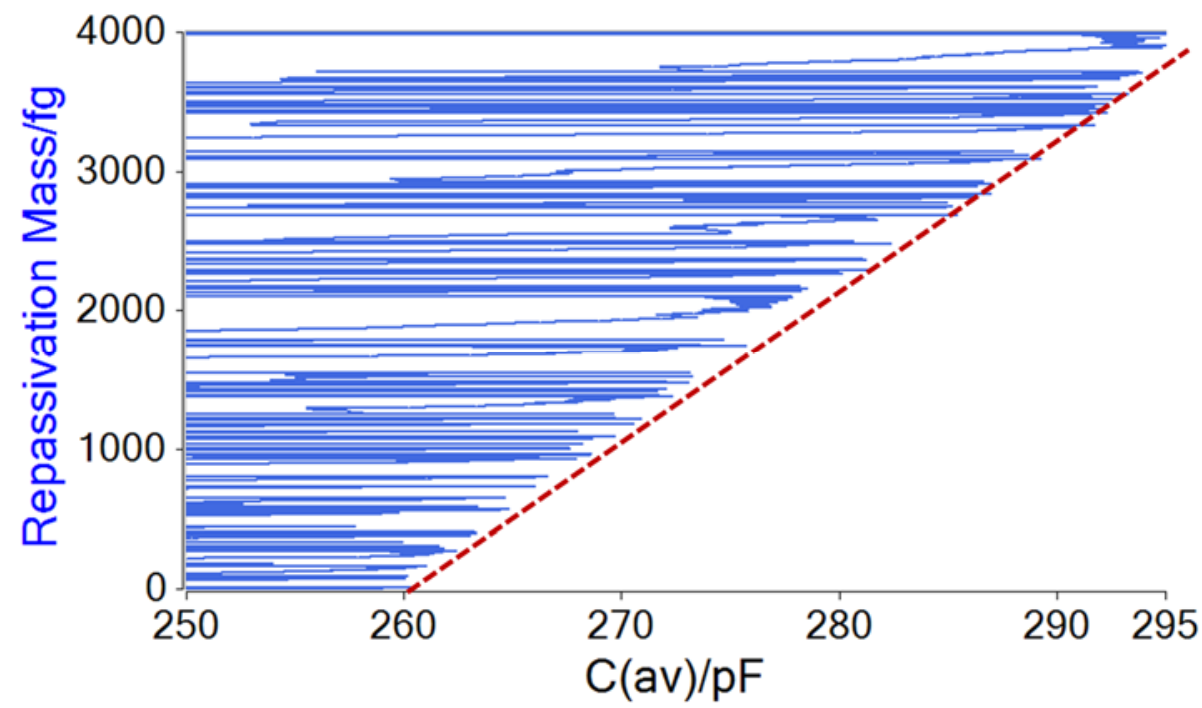

Figure 5. Plot showing the mass change plotted as a function of the electrode capacitance (with a moving point average of 10 points applied to the capacitance data) (-) for a $100 \mu \mathrm{m}$ diameter Al electrode over the complete run. All conditions are as in figure 1 . The red dotted line is a visual aid to illustrate the change in the base capacitance (in the absence of particle/electrode interaction) over the time course of the experiment. The horizontal variation in the capacitance is caused by the changes associated with the particle/electrode interactions. 
However, there is a clear progression to higher apparent electrode capacitance (when particles are not close to the electrode surface) as the regenerated mass increases. The dotted red line (added for visual clarity) shows how the resultant electrode capacitance increases over the course of the experiment. This line has a gradient of $0.12 \mathrm{~g} \mathrm{~F}^{-1}$. This can be used to calculate the rate of change in the surface roughness (and hence the capacitance) as a function of the surface mass regeneration as $8.5 \mathrm{~F} \mathrm{~g}^{-1}$. Presumably, this rate of capacitance change will depend on the materials characteristics (for example, how prone it is to plastic deformation[24,26-28] under the conditions used) and the model used to describe the electrochemical cell. However, further experimental studies are required to validate these assertions. Nevertheless, these measurements show that the method is extremely powerful in assessing the degradation of a surface due to individual particle/surface interactions. In addition, and, critically, this in situ technique possess a high time-resolution without the need for lengthy post treatment and assessment of the surface. This point is important because changes to the interface may occur (through parasitic corrosion for example) which could alter the accuracy of the analysis of the post processed samples. One consequence of this surface damage was that the electrode requires repolishing prior to other data runs (see discussion on errors and the phase angle in the SI data). Lastly, as the surface is exposed to many 100 s of particle impacts per data run (which are not all recorded through the nature of the experimental setup), it is impossible to assign the damage to the surface to an individual or indeed sets of particles. Instead, the electrochemical data must be used to gather useful insight in this area (under the conditions stated).

Combining the high-speed impedance measurements with high-speed imaging of the system, allows individual transients to be associated with individual particles. Figure 6 shows a section of data where three different particles impinged onto the electrode surface. Particle ' 1 ' and ' 2 ' both caused an erosion/corrosion event and a concomitant increase in the effective capacitance of the electrode (see dashed red line). However, particle ' 3 ' did not cause a perceptible erosion/corrosion event or a change in the apparent electrode capacitance (as detected in the electrochemical data). Interestingly the size of particles ' 1 ' and ' 2 ' appear to be similar (300-340 $\mu \mathrm{m}$ largest dimension) compared to ' 3 ' which is $\sim 190 \mu \mathrm{m}$ across. This will result in a reduced impact energy (through its inherent kinetic energy). 


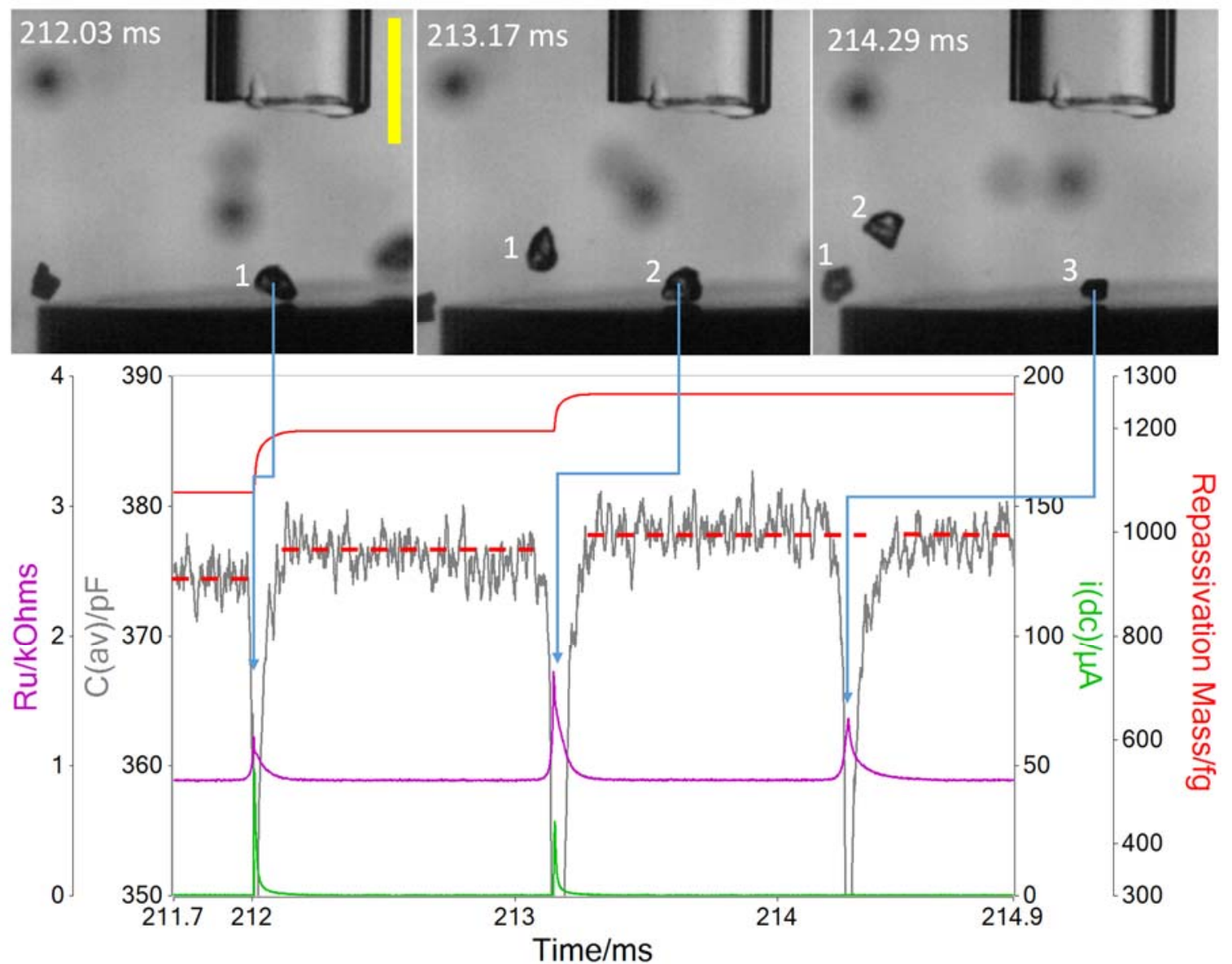

Figure 6. Plot showing the uncompensated resistance $(\mathrm{Ru},-)$ ), capacitance (with a moving point average of 10 points, $\mathrm{C}(\mathrm{av}),-$ ), the faradaic current (i(dc), -) and the mass change (-) for a $100 \mu \mathrm{m}$ diameter Al electrode, (held at $0 \mathrm{~V}$ vs. SS) exposed to sand particle impact in a flowing aerobic electrolyte $\left(0.5 \mathrm{M} \mathrm{Na}_{2} \mathrm{SO}_{4}\right)$ at room temperature. An $800 \mathrm{KHz}\left(200 \mathrm{mV}_{\mathrm{pp}}\right)$ wave was applied to the system. The jet was positioned centrally over the Al electrode in the XY plane, above the electrode, and elevated to $1.5 \mathrm{~mm}$ in the $Z$ axis. Included are the point of impact shots of the three particles (1, 2 and 3 ) with the associated impact time from the start of the data run. The dotted red horizontal line (- -) is a visual aid to show that the capacitance of the electrode has increased for impacts associated with the erosion /corrosion transients (for particles 1,and 2) but remains the same for particle 3. The blue vertical arrows link the images to the associated electrochemical data. The images were recorded at a frame rate of $37.5 \mathrm{kfps}$ and a shutter speed of $20 \mu \mathrm{s}$. The scale bar (upper left image) represents $1 \mathrm{~mm}$.

In addition, the deceleration $[29,30]$ of the particles through the stagnation zone in front of the electrode (shown to change the velocity of a particle from $\sim 5 \mathrm{~m} \mathrm{~s}^{-1}$ at the jet exit to $\sim 1 \mathrm{~m} \mathrm{~s}^{-1}$ on impact under these conditions[13]) may play a role for the smaller particle. The Stokes number is often used to access the effectiveness of a spherical particle to cause indentation. Hunt et al. have suggested a critical Stokes number of $\sim 18$ (for a spherical particle impacting onto aluminium alloys) must be exceeded before indentation effects can be seen[24]. However, the sand particles employed here are far from spherical; hence, a direct comparison is not possible. Nevertheless, comparing the impact point of particle ' 1 ' to particle ' 2 ' is of interest. These particles are of similar size but had notably different impact 
events. Particle ' 1 ' struck the surface at a point, had a smaller resistance and capacitance transient, but caused the most damage while particle ' 2 ' struck the surface (see images in figure 6) in a flatter orientation, causing a larger resistance and capacitance transient but critically a smaller erosion/corrosion transient and subsequent capacitance (surface roughening) change. These 'point of contact' events are of interest as they suggest that the local dynamics at the interface may need to be considered in ascertaining the effect of a specific particle energy on a surface (particularly for these non-spherical cases). Further experiments where the impact velocity, point of impact, size and shape of the particles are assessed in relation to the surface damage needs to be performed before accurate impact energy vs. damage relationships can be quoted with confidence. Other interesting phenomena can be seen in the high-speed imaging data. For example, particle/particle collisions and 'pile ups' occur as the particle 'swarm' interacts with the surface (see SI data). These events cause further tumbling[13] and secondary impacts (see SI data) with the surface, which need further consideration in particle impingement studies, which are beyond the scope of the current work.

\subsection{Conclusions}

The erosion of an interface as the result of single sand particle impingement through a fluid jet has been characterised with a time resolution of $1.25 \mu \mathrm{s}$. Transient increases in the uncompensated resistance are accompanied by decreases in the apparent capacitance as the insulating sand particles approach the surface. The approach of the particle prior to its impact onto the surface and its residence in the local electrochemical environment around the electrode can be detected. Individual erosion/corrosion events can be detected in a particle 'swarm', which involves particle/particle collisions and tumbling. The effect of an individual particle impact on the surface can be detected, as small changes in the apparent electrode capacitance. These changes have been associated with the effective mass regenerated at the electrode surface with an associated rate of roughening documented.

\subsection{Acknowledgements}

The authors would like to thank the University of Southampton for support and funding for MLH and LP and the EPSRC for equipment funding associated with the high-speed camera. 


\section{References}

[1] H.M.I. Clark, On the impact rate and impact energy of particles in a slurry pot erosion tester, Wear. 147 (1991) 165-183. https://doi.org/10.1016/0043-1648(91)90127-G.

[2] D. Aquaro, E. Fontani, Erosion of ductile and brittle materials, Meccanica. 36 (2001) 651-661. https://doi.org/10.1023/A:1016396719711.

[3] J.I. Ukpai, R. Barker, X. Hu, A. Neville, Determination of particle impacts and impact energy in the erosion of X65 carbon steel using acoustic emission technique, Tribol. Int. 65 (2013) 161170. https://doi.org/10.1016/j.triboint.2013.03.012.

[4] L.A. Teran, R.D. Aponte, J. Muñoz-Cubillos, C. V. Roa, J.J. Coronado, J.A. Ladino, F.J. Larrahondo, S.A. Rodríguez, Analysis of economic impact from erosive wear by hard particles in a run-of-the-river hydroelectric plant, Energy. 113 (2016) 1188-1201. https://doi.org/10.1016/j.energy.2016.07.072.

[5] A. Aslam Noon, M.H. Kim, Erosion wear on Francis turbine components due to sediment flow, Wear. 378-379 (2017) 126-135. https://doi.org/10.1016/j.wear.2017.02.040.

[6] R. Elemuren, A. Tamsaki, R. Evitts, I.N.A. Oguocha, G. Kennell, R. Gerspacher, A. Odeshi, Erosion-corrosion of $90^{\circ} \mathrm{AISI} 1018$ steel elbows in potash slurry: Effect of particle concentration on surface roughness, Wear. 430-431 (2019) 37-49. https://doi.org/10.1016/j.wear.2019.04.014.

[7] J. Ryl, J. Wysocka, P. Slepski, K. Darowicki, Instantaneous impedance monitoring of synergistic effect between cavitation erosion and corrosion processes, Electrochim. Acta. 203 (2016) 388-395. https://doi.org/10.1016/j.electacta.2016.01.216.

[8] P.R. Birkin, D.G. Offin, C.J.B. Vian, T.G. Leighton, A.O. Maksimov, Investigation of noninertial cavitation produced by an ultrasonic horn., J. Acoust. Soc. Am. 130 (2011) 3297-308. https://doi.org/10.1121/1.3650537.

[9] I. Hansson, K. a. Mơch, The dynamics of cavity clusters in ultrasonic (vibratory) cavitation erosion, J. Appl. Phys. 51 (1980) 4651. https://doi.org/10.1063/1.328335.

[10] P.R. Birkin, D.G. Offin, T.G. Leighton, The study of surface processes under electrochemical control in the presence of inertial cavitation, Wear. 258 (2005) 623-628. https://doi.org/10.1016/j.wear.2004.05.025.

[11] P.R. Birkin, R. O'Connor, C. Rapple, S.S. Martinez, Electrochemical measurement of erosion from individual cavitation events generated from continuous ultrasound, J. Chem. Soc. Faraday Trans. 94 (1998) 3365-3371.

[12] A.W. Hassel, A.J. Smith, Single particle impact experiments for studying particle induced flow 
corrosion, Corros. Sci. 49 (2007) 231-239. https://doi.org/10.1016/j.corsci.2006.05.021.

[13] P.R. Birkin, J.L. Barber, Particle induced surface erosion - Tumbling and direct impact; a highspeed electrochemical, acoustic and visual study, Wear. 428-429 (2019) 147-153. https://doi.org/10.1016/j.wear.2019.02.011.

[14] K. Sasaki, G.T. Burnstein, Detecting electrochemical transients generated by erosioncorrosion, Electrochim. Acta. 46 (2001) 3675-3683.

[15] S. Silva-Martinez, Applications of Ultrasound in Electrochemistry, University of Southampton, 1997.

[16] S.C. Case, Coalescence of low-viscosity fluids in air, Phys. Rev. E - Stat. Nonlinear, Soft Matter Phys. 79 (2009) 1-10. https://doi.org/10.1103/PhysRevE.79.026307.

[17] S.C. Case, S.R. Nagel, Coalescence in low-viscosity liquids, Phys. Rev. Lett. 100 (2008) 1-4. https://doi.org/10.1103/PhysRevLett.100.084503.

[18] P.R. Birkin, T.M. Foley, J.L. Barber, H.L. Martin, Microsecond resolution of cavitation bubble dynamics using a high-speed electrochemical impedance approach, Chem. Commun. 52 (2016) 11406-11409. https://doi.org/10.1039/c6cc06006d.

[19] R.B. McCleskey, Electrical Conductivity of Electrolytes Found In Natural Waters from (5 to 90) ${ }^{\circ} \mathrm{C}$, J. Chem. Eng. Data. 56 (2011) 317-327. https://doi.org/10.1021/je101012n.

[20] I.M. Abdulagatov, A. Zeinalova, N.D. Azizov, Viscosity of aqueous Na $2 \mathrm{SO} 4$ solutions at temperatures from 298 to $573 \mathrm{~K}$ and at pressures up to $40 \mathrm{MPa}$, Fluid Phase Equilib. 227 (2005) 57-70. https://doi.org/10.1016/j.fluid.2004.10.028.

[21] O. Iulian, F. Sǐrbu, C. Stoicescu, Density and apparent molar volume prediction in some ternary electrolyte solutions, Rev. Roum. Chim. 53 (2008) 1125-1129.

[22] H. Bouazaze, J. Fransaer, F. Huet, P. Rousseau, V. Vivier, Electrolyte-resistance change due to an insulating sphere in contact with a disk electrode, Electrochim. Acta. 55 (2010) 1645-1655. https://doi.org/10.1016/j.electacta.2009.10.043.

[23] Z. Gao, C. Wang, Y. Liu, Evaluation of cavitation erosion-corrosion process of Q235 and ZG06Cr13Ni4Mo with various applied potentials, Int. J. Electrochem. Sci. 10 (2015) 64876499.

[24] A. Ruiz-Angulo, S. Roshankhah, M.L. Hunt, Surface deformation and rebound for normal single-particle collisions in a surrounding fluid, J. Fluid Mech. 871 (2019) 1044-1066. https://doi.org/10.1017/jfm.2019.349.

[25] Z.B. Zheng, Y.G. Zheng, X. Zhou, S.Y. He, W.H. Sun, J.Q. Wang, Determination of the critical flow velocities for erosion-corrosion of passive materials under impingement by $\mathrm{NaCl}$ solution containing sand, Corros. Sci. 88 (2014) 187-196. 
https://doi.org/10.1016/j.corsci.2014.07.043.

[26] G.G. Joseph, R. Zenit, M.L. Hunt, A.M. Rosenwinkel, Particle-wall collisions in a viscous fluid, J. Fluid Mech. 433 (2001) 329-346. https://doi.org/10.1017/S0022112001003470.

[27] G. Joseph, Collisional dynamics of macroscopic particles in a viscous fluid, PhD Thesis, Calif. Inst. Technol. (2003) 119.

[28] A. Ruiz-Angulo, M.L. Hunt, Measurements of the coefficient of restitution for particle collisions with ductile surfaces in a liquid, Granul. Matter. 12 (2010) 185-191. https://doi.org/10.1007/s10035-010-0166-y.

[29] M. Parsi, K. Najmi, F. Najafifard, S. Hassani, B.S. McLaury, S.A. Shirazi, A comprehensive review of solid particle erosion modeling for oil and gas wells and pipelines applications, J. Nat. Gas Sci. Eng. 21 (2014) 850-873. https://doi.org/10.1016/j.jngse.2014.10.001.

[30] Y. Zhang, E.P. Reuterfors, B.S. McLaury, S.A. Shirazi, E.F. Rybicki, Comparison of computed and measured particle velocities and erosion in water and air flows, Wear. 263 (2007) 330338. https://doi.org/10.1016/j.wear.2006.12.048. 